\title{
X-ray variability of 104 active galactic nuclei
}

\author{
O. González-Martín ${ }^{1,2,3} \dagger$ and S. Vaughan ${ }^{4}$
}

${ }^{1}$ Instituto de Astrofísica de Canarias (IAC), C/Vía Láctea, s/n, E-38205 La Laguna, Spain

${ }^{2}$ Departamento de Astrofísica, Universidad de La Laguna (ULL), E-38205 La Laguna, Spain

${ }^{3}$ Instituto de Astrofísica de Andalucía (CSIC), Glorieta de la Astronomía, s/n 18008 Granada, Spain

${ }^{4}$ Department of Physics and Astronomy, Leicester University, Leicester LE1 7RH, UK email: omairagm@iac.es

\begin{abstract}
We have performed a uniform analysis of the power spectrum densities (PSDs) of 104 nearby $(\mathrm{z}<0.4)$ active galactic nuclei $(\mathrm{AGN})$ using 209 XMM-Newton/pn observations, including several AGN classes. These PSDs span $\simeq 3$ decades in temporal frequencies, ranging from minutes to days. We have fitted each PSD to two models: (1) a single power-law model and (2) a bending power-law model. A fraction of $72 \%$ show significant variability. The PSD of the majority of the variable AGN was well described by a simple power-law with a mean index of $\alpha=2.01 \pm 0.01$. In 15 sources we found that the bending power law model was preferred with a mean slope of $\alpha=3.08 \pm 0.04$ and a mean bend frequency of $\left\langle\nu_{b}\right\rangle \simeq 2 \times 10^{-4} \mathrm{~Hz}$. Only KUG 1031+398 (RE J1034+396) shows evidence for quasi-periodic oscillations. The 'fundamental plane' relating variability timescale, black hole mass, and luminosity is demonstrated using the new X-ray timing results presented here together with a compilation of the previously detected timescales from the literature.
\end{abstract}

Keywords. Accretion, accretion disks - Galaxies:active - Galaxies:nuclei - X-ray:galaxies

\section{Introduction}

Active galactic nuclei (AGN), powered by accretion onto supermassive black holes (SMBHs), are thought to be scaled up versions of Galactic black hole X-ray binaries (BH-XRBs). X-ray variability - thought to originate in the innermost regions of the accretion flow - is an important aspect of the AGN-XRB connection (Uttley et al. 2002; Markowitz et al. 2003; McHardy et al. 2006; Vaughan et al. 2011). Both AGN and BHXRBs show 'red noise' power spectra (or power spectral density, PSD) that decrease steeply at high frequencies (short timescales) as a power law, $P(\nu) \sim \nu^{-\alpha}$ (where $\nu$ is temporal frequency), typically with $\alpha \approx 2$. Below some characteristic frequency $\nu_{b}$ the PSDs flatten, and these bend frequencies scale approximately inversely with the BH mass from BH-XRBs to AGN. However, the PSDs of BH-XRBs depend on the 'state' in which the system is observed. In the soft state the PSD is usually well described by a simple bending power-law, with a slope $\alpha>2$ above some bend frequency $\nu_{\mathrm{b}}$, and a slope of $\alpha \approx 1$ extending unbroken to much lower frequencies (Cui et al. 1997). In the hard state the PSDs are generally more complex, with additional bends and peaks at lower frequencies, and are usually modeled as a mixture of zero-centred Lorentzian components (Remillard \& McClintock 2006). One striking and common property of the $\mathrm{X}$-ray variability of $\mathrm{BH}-\mathrm{XRBs}$ is the prominent quasi-periodic oscillations (QPOs), which are seen as strong, narrow peaks in the PSDs (Remillard \& McClintock 2006). Until recently there were no robust claims of QPOs in AGN (Vaughan \& Uttley 2005). This changed with the reported QPO for the Narrow-line Seyfert 1 (NLSy1) RE J1034+396, which remains the only case to date for an AGN (Gierliński et al. 2008).

\section{$\dagger$ Juan de la Cierva fellow}


The main purpose for the present analysis is to characterize the PSD of a large sample of AGN including a range of AGN subclasses. With this large sample we address some outstanding problems such as: what is the most common PSD shape for AGN? how does this compare to BH-XRBs? How does the PSD vary with AGN properties (BH mass, AGN subtype or luminosity)? Are there any other strong candidate of QPO among the AGN? We have made use of the extensive XMM-Newton archive to obtain a sample of 104 AGN with 209 observations of at least $40 \mathrm{ksec}$. This is the largest sample of PSDs of AGN ever analyzed.

\section{Sample}

We have selected observations of AGN from the XMM-Newton public archives until February 2012. The AGN are all identified in the catalogue of Quasars and AGN by Véron-Cetty \& Véron $(2010) \dagger$ with $z<0.4$. We have selected data according to the following inclusion criteria: (1) include one or more members of the AGN sample (search radius 9 arcmin from the centre of the FOV), and (2) observation duration $T>40 \mathrm{ksec}$. The second criteria ensures a reasonable frequency range for the PSD estimation (the lowest observable frequency scales is $1 / T$ ). The final sample comprises 209 observations and 104 distinct AGN.

\section{PSD analysis}

The PSD gives the distribution of variability power (amplitude squared) as a function of the temporal frequency. The standard method for estimating the PSD is by calculating the periodogram (Priestley 1981; Vaughan et al. 2003A). The periodogram data were fitted using the maximum likelihood method discussed in Vaughan (2010) and Barret \& Vaughan (2012). Following the previous work on the PSDs of AGN we used two models:

- Model A: simple power law plus constant. The model has three parameters: $N$, the power law normalization; $\alpha$, the spectral index; $C$, an additive constant to account for Poisson noise.

- Model B: a bending power law plus constant. The free parameters for this model are: the normalization $N$, the spectral index above the bend $\alpha$, the bend frequency $\nu_{b}$, and the constant $C$.

These $X M M-N e w t o n$ data are relatively insensitive to the exact low frequency index, and we assume the typical value of 1 found from long-term X-ray monitoring studies (e.g. Uttley et al. 2002; Markowitz et al. 2003; McHardy et al. 2006). Temporally close observations of the same objects were analyzed together to better constrain the final parameters. The Likelihood Ratio Test (LRT) was used to select between the two models. The simpler model (A) is preferred in the absence of a strong preference for the more complex model (B). As a crude but simple check for the presence of highly coherent oscillations (strictly or quasi-periodic) we identify the largest data/model outlier from each periodogram. This was then compared to the $\chi_{r}^{2}$ distribution expected for periodogram data to give a $p$-value. Candidate QPOs were flagged when a $p<0.01$ criterion was met (after correcting for the number of frequencies in the periodogram).

\section{PSD shape}

The PSDs of the majority of the variable AGN could be described by a single powerlaw (i.e. Model A) with a mean slope of $\alpha \approx 2$. In 17 sources - all Type- 1 Seyferts (many

\footnotetext{
$\dagger$ http://heasarc.nasa.gov/W3Browse/all/veroncat. html
} 

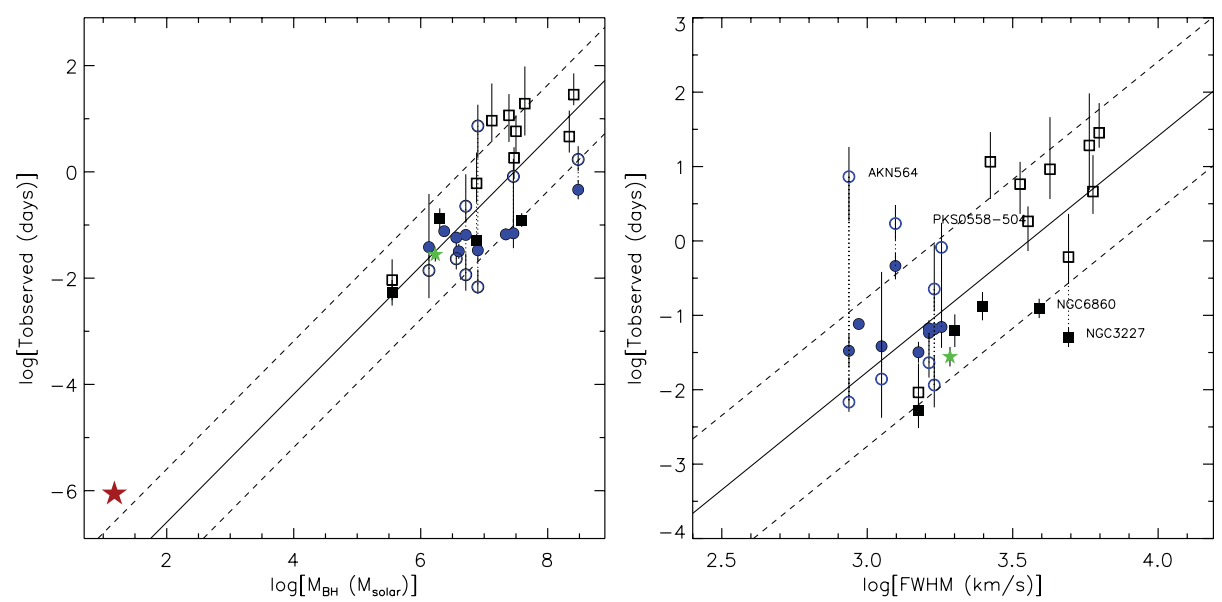

Figure 1. (left): Observed bend timescale versus the BH mass. The continuous line is the best-fitting following eqn. 6.1 . The dashed lines illustrate the \pm 1 dex region around this model. Circles represent NLSy1s, squares represent Type-1 Seyferts, and the small green star is the Type-2 Seyfert. The open symbols are data-points reported in the literature and filled symbols are the data-points reported here. The Cygnus X-1 data are shown as a red big star. A dotted line is used to link multiple frequency bends for the same object. (right): Observed characteristic timescale versus the FWHM of $\mathrm{H}_{\beta}$ expressed in $\mathrm{km} \mathrm{s}^{-1}$.

are NLSy1s) but the Circinus galaxy $\ddagger$ - we found a strong preference for the bending power-law model (i.e. Model B). For this subset, the slope above the bend frequency is steep, $\alpha \sim 3.0$, above a bend frequency typically around $\nu_{b} \sim 3.4 \times 10^{-4} \mathrm{~Hz}$. Spectral leakage, which can bias the slope estimates in some cases, does not appear to explain discrepancy between the slopes found for Model A and B.

In order to study whether we detect all the expected bends, we have computed the predicted $\dagger$ characteristic time scale $T_{b}$ for sample members with $M_{B H}$ estimates (70 out of the 104), assuming bolometric luminosity of $L_{b o l}=30 \times L_{2-10}$. The PSD bend timescales is predicted to be within the observed frequency range for only 17 sources. Of these we do indeed detect bends in 13. For the rest of the sample members the predicted $T_{b}$ value places it outside of the observed PSD bandpass. Moreover, all the non-variable sources have an expected bend well above the range analyzed here. Therefore, the overall PSD shape of AGN is consistent with BH-XRBs, although the slopes at frequencies above the bend are steeper than expected in BH-XRBs.

\section{QPO detections}

We find no strong evidence for highly coherent oscillations (QPOs) beyond the well known example of KUG 1031+398. Some authors have pointed to the extremely soft spectrum of this source as an explanation of why this source appears to be unique in showing a QPO. In fact, even for this source the QPO appears to be transient (Middleton et al. 2009). Less coherent oscillations, i.e. QPOs with a broader features in the PSD, are common to $\mathrm{BH}-\mathrm{XRBs}$, especially in harder spectral states, but are difficult to detect in the majority of the currently available data, as discussed by Vaughan \& Uttley (2005) and Vaughan et al. (2011).

$\ddagger$ Note that the Circinus galaxy contains a very bright Ultraluminous X-ray source that it is demonstrated to be the responsible for the QPO observed in this galaxy.

$\dagger$ We have estimated characteristic time scale assuming the relation found by McHardy et al. (2006) 


\section{Scaling relations of the characteristic time-scale}

Several papers have demonstrated a strong, approximately linear correlation between the PSD bend timescale $\left(T_{b}=1 / \nu_{b}\right)$ and the BH mass (e.g. Uttley et al. 2002; Markowitz et al. 2003). The simplest relation between timescale and fundamental AGN properties that we might consider is

$$
\log \left(T_{b}\right)=A \log \left(M_{B H}\right)+C
$$

where $A$ and $C$ are coefficients, and we expect $A \approx 1$ for a linear mass-timescale relation. McHardy et al. (2006) presented an extension of this model allowing for dependence of $T_{b}$ on both $M_{B H}$ and bolometric luminosity $L_{b o l}$ :

$$
\log \left(T_{b}\right)=A \log \left(M_{B H}\right)+B \log \left(L_{b o l}\right)+C
$$

We fitted models following eqn. 6.1 and 6.2 to data from 22 AGN (including objects with frequency bends already reported in the literature). The models were fitted by simple linear regression (i.e. unweighted least squares) on the log transformed variables, with $T_{b}$ in units of days, $L_{b o l}$ in units of $10^{44} \mathrm{erg} \mathrm{s}^{-1}$, and $M_{B H}$ in units of $10^{6} \mathrm{M}_{\odot}$, as in McHardy et al. (2006). Fitting equation 6.1 gave parameter estimates $A=1.09 \pm 0.21$ and $C=-1.70 \pm 0.29$, leaving a sum squared error (SSE) of 11.14 (for 19 degrees of freedom, dof). The data and best-fitting regression line are shown in Fig. 1 (left). Fitting equation 6.2 gave parameter estimates $A=1.34 \pm 0.36, B=-0.24 \pm 0.28$, and $C=-1.88 \pm 0.36$, leaving a sum squared error (SSE) of 10.69 (for 18 dof). The parameter governing the luminosity dependence, $B$, is consistent with zero $(\mathrm{p}=0.38$ for 18 dof $)$.

In order to test how well these scaling relations work over the full range of black hole masses we also show representatives values for the BH-XRB Cygnus X-1 (red star in Fig. 1, left). The Cygnus X-1 points were not included in the fitting, yet are clearly consistent with an extrapolation to much lower $M_{B H}$, strongly supporting the reliability of such relations over the full range of $M_{B H}$. Indeed, fitting the two models including the Cygnus $\mathrm{X}-1$ data resulted in parameter estimates consistent with those given above.

The main difference compared with the results obtained by McHardy et al. (2006) is a weak dependence of $T_{b}$ on $L_{b o l}$ in the present analysis. This weak dependence on $L_{b o l}$ could be due to either the fact that our sample is more complete or due to uncertainties on the $\mathrm{BH}$ mass and/or $\mathrm{L}_{\mathrm{bol}}$ estimates. A bigger sample with better estimates on the $\mathrm{BH}$ mass and $\mathrm{L}_{\mathrm{bol}}$ is need to check the dependence on $\mathrm{L}_{\mathrm{bol}}$ and determine the parameters of the so-called 'fundamental plane' of black hole X-ray variability.

\section{References}

Barret, D. \& Vaughan, S., 2012 ApJ, in press (arXiv:1112.0535)

Cui, W., Zhang, S. N., Focke, W., \& Swank, J. H. 1997, ApJ, 484, 383

Gierliński, M., Middleton, M., Ward, M., \& Done, C. 2008, Nature, 455, 369

Markowitz, A., et al. 2003, ApJ, 593, 96

McHardy, I. M., Koerding, E., Knigge, C., Uttley, P., \& Fender, R. P. 2006, Nature, 444, 730

Middleton, M., Done, C., Ward, M., Gierliński, M., \& Schurch, N. 2009, MNRAS, 394, 250

Priestley, M. B. 1981, Spectral Analysis and Time Series, Academic Press, London.

Remillard, R. A. \& McClintock, J. E. 2006, ARAA, 44, 49

Uttley, P., McHardy, I. M., \& Papadakis, I. E. 2002, MNRAS, 332, 231

Vaughan, S., Edelson, R., Warwick, R. S., \& Uttley, P. 2003A, MNRAS, 345, 1271

Vaughan, S. \& Uttley P., 2005, MNRAS, 362, 235

Vaughan, S. 2010, MNRAS, 402, 307

Vaughan, S., Uttley, P., Pounds, et al. 2011, MNRAS, 413, 2489

Véron-Cetty, M.-P. \& Véron, P. 2010, A\&A, 518, A10 